\title{
Therapeutic hypothermia for neonatal hypoxic ischaemic encephalopathy should not be discontinued in low- and middle-income countries
}

Perinatal asphyxia is a major cause of death and disability in children. Therapeutic hypothermia (TH) has become a standard of care for newborn infants who have sustained hypoxic ischaemic encephalopathy (HIE) due to perinatal asphyxia. There is compelling evidence to support this approach. A Cochrane systematic review of 11 prospective randomised controlled trials including 1505 newborns showed that TH started within 6 hours of birth in infants with HIE significantly decreased mortality and neurodevelopmental disability in survivors. ${ }^{[1]}$ Several academic hospitals in South Africa (SA) have been cooling newborns with HIE since 2008, with favourable outcomes. ${ }^{[2]}$ However, Thayyil et al. ${ }^{[3]}$ recently published results of the HELIX trial, a large, well-designed, multicentre randomised controlled trial conducted in India, Sri Lanka and Bangladesh, which showed that TH did not decrease neurodevelopmental handicap and actually increased mortality. The authors of this study concluded that TH should not be offered in the treatment of neonatal encephalopathy in low- and middle-income countries (LMICs). The HELIX study was conducted using servo-controlled cooling equipment in hospitals with intensive care facilities, so the increased mortality could not be explained by inferior equipment or lack of intensive care facilities. The HELIX study group subsequently wrote a commentary in the Indian Journal of Pediatrics stating that it is time to stop offering TH for neonatal encephalopathy in LMICS, owing to the lack of benefit and increased mortality. ${ }^{[4]}$ These authors argue that the body of research supporting the use of TH was performed in high-income countries (HICs) and that the HELIX study results conclusively show that it is of no benefit in LMICs.

We believe for several reasons that it is inappropriate for the HELIX authors to make a generalisation that TH should not be used in LMICs based on the findings of their study. Firstly, it is not clear that the patients enrolled in the HELIX trial had sustained acute intrapartum hypoxia, for which $\mathrm{TH}$ has been shown to be effective. Sentinel events suggestive of intrapartum hypoxia and fetal heart rate decelerations were only reported in $11 \%$ and $6 \%$ of enrolled patients, respectively. Only $3 \%$ of mothers were reported to have had a prolonged second stage, and $1 \%$ had obstructed labour. There was no report of neonatal blood gases within the first hour after delivery, and only $11 \%$ had cord blood gases documented. These numbers are much lower than those that have been reported in studies that have shown TH to be effective. Secondly, it would appear that the HELIX group enrolled neonates who were critically ill compared with other studies, with $80 \%$ and $70 \%$ of their patients requiring inotropes and mechanical ventilation, respectively, compared with $34 \%$ and $17 \%$ from an SA study. This could explain their high mortality of $36 \%$ compared with mortality of $14 \%$ in SA studies. ${ }^{[5]}$ Thirdly, basal ganglia injury on brain magnetic resonance imaging, which is an injury related to an acute intrapartum hypoxic event, was only seen in $25 \%$ of neonates in the HELIX trial compared with $48-79 \%$ in studies in HICs where TH was reported to be effective ${ }^{[6,7]}$ Lastly, the results from TH trials in HICs reported a $47 \%$ reduction in disabling cerebral palsy in neonates who received $\mathrm{TH}$ compared with those who received the standard of care.

We have been offering TH in neonates with HIE in SA for more than a decade, with apparently reasonable outcomes, with in-hospital mortality of $13-14 \%^{[2,5]}$ and normal neurological outcome or mild impairment in $82 \%$ at 12 months ${ }^{[2]}$ and $68 \%$ at $18-24$ months. ${ }^{[8]}$ We therefore have a major concern with this 'all or none' approach recommended by the HELIX authors. In addition, contrary to what the HELIX authors have recommended, a recent meta-analysis on TH for asphyxiated neonates with encephalopathy recommends that TH should be offered as part of the standard of care, as the reduction in mortality was found to be greatest in LMICs. ${ }^{[9]}$ Aneja and Sharma ${ }^{[10]}$ propose a more measured approach and acknowledge the strengths of the HELIX study, but also discuss several possible reasons for the unexpected poor outcomes with $\mathrm{TH}$, rather than the location being LMICs per se. In addition to the reasons highlighted above, these include a high number of small-for-gestational-age and low-birthweight infants in the HELIX trial, a significant number of infants born outside hospital, and a large proportion of infants with seizures at the time of enrolment. In addition, the HELIX authors themselves suggest that population factors may account for the different response to $\mathrm{TH}$ in their study. ${ }^{[3]}$

We therefore suggest a moderated response to the HELIX trial results. We still do not understand everything about perinatal asphyxia and HIE, and it is premature to dismiss a potentially beneficial therapy based on the results of a single study conducted in a population and context very different from our own. As suggested by Aneja and Sharma, ${ }^{[10]} \mathrm{TH}$ should not be regarded as a standard of care, but should be used in referral hospitals and according to strict protocols. We need to continue research into HIE and TH to identify those infants who will benefit the most from this treatment. The Neonatal Encephalopathy with Suspected Encephalopathy (NESHIE) study is an ongoing prospective multicentre observational study in SA to determine the role of genomics and other factors in HIE. We may need to limit TH to certain subgroups of infants. For example, it appears that out-born babies may not have as favourable outcomes and should therefore possibly be excluded from $\mathrm{TH}$.

Randomised controlled trials (RCTs) are the gold standard for evaluating the effectiveness of interventions, but since $\mathrm{TH}$ has previously been shown to be effective and safe in reducing adverse HIE outcomes (in both LMICs and HICs) and we have been offering $\mathrm{TH}$ for the past 10 years, it has so far not been ethical or appropriate to randomise asphyxiated neonates into groups with or without TH. Perhaps the HELIX results will allow ethical approval for a prospective, multicentre RCT of TH in SA to properly evaluate $\mathrm{TH}$ in the local context, allowing us to make more informed decisions about its ongoing use in our centres. It could also be more generalisable to us and our neighbouring countries than perhaps the HELIX trial is.

In conclusion, although the results of the HELIX trial are concerning and highlight the need for caution, we do not support the notion of abandoning the use of TH in SA. Instead, we agree that TH should be used according to strict protocols in referral hospitals. We also support ongoing research into both HIE and TH in our local context, and would welcome the opportunity to perform an RCT.

Daynia E Ballot, Tanusha D Ramdin, Rossella M Bandini Department of Paediatrics and Child Health, Charlotte Maxeke Johannesburg Academic Hospital and Faculty of Health Sciences, University of the Witwatersrand, Johannesburg, South Africa daynia.ballot@wits.ac.za 
Firdose Nakwa, Sithembiso Velaphi, Melantha Coetzee

Department of Paediatrics and Child Health, Chris Hani Baragwanath Academic Hospital and Faculty of Health Sciences, University of the Witwatersrand, Johannesburg, South Africa

\section{Khomotso Masemola}

Department of Paediatrics and Child Health, Kalafong Hospital and Faculty of Health Sciences, University of Pretoria, South Africa

\section{Gugulabatembunamahlubi J D Kali}

Department of Paediatrics and Child Health, Tygerberg Hospital and Faculty of Medicine and Health Sciences, Stellenbosch University, Cape Town, South Africa

\section{Alan R Horn, Shakti Pillay}

Department of Paediatrics and Child Health, Groote Schuur Hospital and Faculty of Health Sciences, University of Cape Town, South Africa

\section{Jeanne van Rensburg, Michael S Pepper}

Institute for Cellular and Molecular Medicine, Faculty of Health Sciences, University of Pretoria, South Africa
1. Jacobs SB, Berg M, Hunt R, Tarnow-Mordi WO, Inder TE, Davis PG. Cooling for newborns with hypoxic ischaemic encephalopathy. Cochrane Database Syst Rev 2013, Issue 1. Art. No.: CD003311. https://doi. rg/10.1002/14651858.CD003311.pub3

2. Kali GT, Martinez-Biarge M, van Zyl J, Smith J, Rutherford M. TH for neonatal hypoxic-ischaemic encephalopathy had favourable outcomes at a referral hospital in a middle-income country. Acta Paediatr 2016;105(7):806-815. https://doi.org/10.1111/apa.13392

3. Thayyil S, Pant S, Montaldo P, et al. Hypothermia for moderate or severe neonatal encephalopathy in low-income and middle-income countries (HELIX): A randomised controlled trial in India, Sri Lanka, and Bangladesh. Lancet Glob Health 2021;9(9):e1273-e1285. https://oi.org/10.1016/S2214$109 \mathrm{X}(21) 00264-3$

4. Krishnan V, Kumar V, Shankaran S, Thayyil S. Rise and fall of TH in low-resource settings: Lessons from the HELIX trial. Indian J Pediatr 2021 (epub 23 July 2021). https://doi.org/10.1007/s12098-021-03861-y 5adayachee N, Ballot DE. Outcomes of neonates with perinatal asphyxia at a tertiary academic hospital in Johannesburg, South Africa. S Afr J Child Health 2013;7(3):89-94. https://doi.org/10.7196/SAJCH.574 6. Azzopardi D, Brocklehurst P, Edwards D, et al. The TOBY Study: Whole body hypothermia for the treatment of perinatal asphyxial encephalopathy: A randomised controlled trial. BMC Pediatr 2008:8:17. https://doi.org/10.1186/1471-2431-8-17 Shankaran S, Laptook AR, Ehrenkranz RA, et al. Whole-body hypothermia for neonates with hypoxic-ischemic encephalopathy. N Engl J Med 2005;353(15):1574-1584. https://doi.org/10.1056/ hypoxic-ischem

8. Mbatha S, Nakwa FL, Thandrayen K, Velaphi S. Neurodevelopmental outcome in neonates with hypoxicischaemic encephalopathy managed with TH in a tertiary-level public hospital outside an intensive care unit setting. Paediatr Int Child Health 2021 (epub 8 September 2021). https://doi.org/10.1080/2046904 7.2021 .1967625

9. Abate BB, Bimerew M, Gebremichael B, et al. Effects of TH on death among asphyxiated neonates with hypoxic-ischemic encephalopathy: A systematic review and meta-analysis of randomized control trials. PLoS ONE 2021;16(2):e0247229. https://doi.org/10.1371/journal.pone.0247229

10. Aneja S, Sharma S. Hypoxic ischaemic encephalopathy in low resource settings - time to stop cooling? Lancet Glob Health 2021;9(9):el187-e1 188. https://doi.org/10.1016/S2214-109X(21)00343-0

S Afr Med J 2021;111(12):1168-1169. https://doi.org/10.7196/SAMJ.2021.v111i12.16180 